\title{
Sustained Effectiveness of Pfizer-BioNTech and Moderna Vaccines Against COVID-19 Associated Hospitalizations Among Adults — United States, March-July 2021
}

\begin{abstract}
Mark W. Tenforde, MD, PhD ${ }^{1, *}$; Wesley H. Self, MD²,*; Eric A. Naioti, MPH ${ }^{1}$; Adit A. Ginde, MD 3 ; David J. Douin, MD ${ }^{3}$; Samantha M. Olson, MPH ${ }^{1}$; H. Keipp Talbot, $\mathrm{MD}^{2}$; Jonathan D. Casey, $\mathrm{MD}^{2}$; Nicholas M. Mohr, MD4; Anne Zepeski, PharmD ${ }^{4}$; Manjusha Gaglani, MBBS ${ }^{5,6}$; Tresa McNeal, MD;

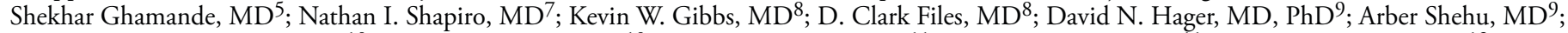
Matthew E. Prekker, MD ${ }^{10}$; Heidi L. Erickson, MD ${ }^{10}$; Michelle N. Gong, MD ${ }^{11}$; Amira Mohamed, MD ${ }^{11}$; Daniel J. Henning, MD ${ }^{12}$;

Jay S. Steingrub, MD ${ }^{13}$; Ithan D. Peltan, MD ${ }^{14}$; Samuel M. Brown, MD ${ }^{14}$; Emily T. Martin, PhD ${ }^{15}$; Arnold S. Monto, MD ${ }^{15}$; Akram Khan, MD ${ }^{16}$;

Catherine L. Hough, MD ${ }^{16}$; Laurence W. Busse, MD ${ }^{17}$; Caitlin C. ten Lohuis, ACNP-BC ${ }^{17}$; Abhijit Duggal, MD ${ }^{18}$; Jennifer G. Wilson, MD ${ }^{19}$; Alexandra June Gordon, MD ${ }^{19}$; Nida Qadir, MD ${ }^{20}$; Steven Y. Chang, MD, PhD ${ }^{20}$; Christopher Mallow, MD ${ }^{21}$; Carolina Rivas ${ }^{21}$;

Hilary M. Babcock, MD²2; Jennie H. Kwon, DO ${ }^{22}$; Matthew C. Exline, MD²3; Natasha Halasa, $\mathrm{MD}^{2}$; James D. Chappell, MD, PhD²;

Adam S. Lauring, MD, PhD ${ }^{24}$; Carlos G. Grijalva, MD²; Todd W. Rice, $\mathrm{MD}^{2}$; Ian D. Jones, $\mathrm{MD}^{2}$; William B. Stubblefield, MD²; Adrienne Baughman²; Kelsey N. Womack, $\mathrm{PhD}^{2}$; Christopher J. Lindsell, $\mathrm{PhD}^{2}$; Kimberly W. Hart, MA²; Yuwei Zhu, $\mathrm{MD}^{2}$; Meagan Stephenson, $\mathrm{MPH}^{1}$; Stephanie J. Schrag, DPhil ${ }^{1}$; Miwako Kobayashi, MD ${ }^{1}$; Jennifer R. Verani, MD ${ }^{1}$; Manish M. Patel, MD ${ }^{1}$; IVY Network Investigators
\end{abstract}

On August 18, 2021, this report was posted as an MMWR Early Release on the MMWR website (https://www.cdc.gov/mmwr).

Real-world evaluations have demonstrated high effectiveness of vaccines against COVID-19-associated hospitalizations (1-4) measured shortly after vaccination; longer follow-up is needed to assess durability of protection. In an evaluation at 21 hospitals in 18 states, the duration of mRNA vaccine (Pfizer-BioNTech or Moderna) effectiveness (VE) against COVID-19-associated hospitalizations was assessed among adults aged $\geq 18$ years. Among 3,089 hospitalized adults (including 1,194 COVID-19 case-patients and 1,895 nonCOVID-19 control-patients), the median age was 59 years, $48.7 \%$ were female, and $21.1 \%$ had an immunocompromising condition. Overall, $141(11.8 \%)$ case-patients and 988 $(52.1 \%)$ controls were fully vaccinated (defined as receipt of the second dose of Pfizer-BioNTech or Moderna mRNA COVID-19 vaccines $\geq 14$ days before illness onset), with a median interval of 65 days (range $=14-166$ days) after receipt of second dose. VE against COVID-19-associated hospitalization during the full surveillance period was $86 \%$ (95\% confidence interval $[\mathrm{CI}]=82 \%-88 \%$ ) overall and $90 \%$ (95\% CI $=87 \%-92 \%)$ among adults without immunocompromising conditions. VE against COVID-19- associated hospitalization was $86 \%(95 \% \mathrm{CI}=82 \%-90 \%) 2-12$ weeks and $84 \%(95 \% \mathrm{CI}=77 \%-90 \%) 13-24$ weeks from receipt of the second vaccine dose, with no significant change between these periods $(\mathrm{p}=0.854)$. Whole genome sequencing of 454 case-patient specimens found that $242(53.3 \%)$ belonged to the B.1.1.7 (Alpha) lineage and 74 (16.3\%) to the B.1.617.2 (Delta) lineage. Effectiveness of mRNA vaccines against COVID-19-associated hospitalization was

\footnotetext{
*These authors contributed equally to this report.
}

sustained over a 24-week period, including among groups at higher risk for severe COVID-19; ongoing monitoring is needed as new SARS-CoV-2 variants emerge. To reduce their risk for hospitalization, all eligible persons should be offered COVID-19 vaccination.

Evaluations of authorized mRNA COVID-19 vaccines (Pfizer-BioNTech and Moderna) have consistently demonstrated high VE across diverse populations (1,5). Because COVID-19 vaccines were initially authorized in the United States in December 2020, evaluations of real-world effectiveness have been subject to a short period of postvaccination follow-up. Monitoring durability of protection after COVID-19 vaccination can help determine whether booster vaccines might be indicated, particularly with continued emergence of new variants that might overcome vaccine-induced immunity. In real-world settings, durability of protection has commonly been measured by comparing the odds of vaccination in laboratory-confirmed case-patients and control-patients who tested negative for infection, by time since vaccination $(6,7)$.

During March 11-July 14, 2021, adults aged $\geq 18$ years admitted to 21 hospitals in 18 states were included in an analysis of durability of vaccine-induced protection. Casepatients had COVID-19-like illness ${ }^{\dagger}$ and had received a positive SARS-CoV-2 reverse transcription-polymerase chain reaction (RT-PCR) or antigen test result. A first group of hospital-based control-patients had COVID-19-like illness and had negative SARS-COV-2 results by all tests, including at least one RT-PCR test. A second hospital-based control group of patients without COVID-19-like illness (and therefore unlikely to be hospitalized for COVID-19-like illness)

\footnotetext{
$\dagger^{\dagger}$ COVID-19-like illness was defined as having one or more of the following: fever, cough, shortness of breath, loss of taste, loss of smell, use of respiratory support for the acute illness, or new pulmonary findings on chest imaging consistent with pneumonia.
} 
was also enrolled (4). This second control group also received negative SARS-CoV-2 results by all tests, including at least one RT-PCR test. Eligibility for enrollment as a case-patient or one of these controls required SARS-CoV-2 testing within 10 days of symptom onset and hospital admission within 14 days of symptom onset. Final case/control status was determined using clinical testing results and central laboratory RT-PCR testing of upper respiratory specimens (nasal swabs or saliva) performed at a central laboratory (Vanderbilt University Medical Center, Nashville, Tennessee) (4). Specimens positive for SARS-CoV-2 with cycle threshold values $<32$ were sent to University of Michigan (Ann Arbor, Michigan) for whole genome sequencing and SARS-CoV-2 lineage determination (4).

Patients or their proxies were interviewed about baseline demographic characteristics, clinical history (including COVID-19-like signs or symptoms experienced and date of illness onset), and history of COVID-19 vaccination. Vaccine was considered to have been administered if vaccination dates and product names were verified through medical records, state immunization registries, vaccination record cards, or provider or pharmacy records, or if plausibly reported by patient or proxy with date and location of vaccination. A patient was considered to be fully vaccinated if both doses of an authorized mRNA COVID-19 vaccine were administered, with the second dose received $\geq 14$ days before illness onset. ${ }^{\S}$ Participants were excluded from this analysis if they received only 1 dose of an mRNA COVID-19 vaccine, received 2 doses with the second dose $<14$ days before illness onset, received a non-mRNA COVID-19 vaccine, or received mixed products of an mRNA COVID-19 vaccine (i.e., a different product for each dose).

Vaccine effectiveness against COVID-19-associated hospitalization was estimated using logistic regression, comparing the odds of being fully vaccinated versus unvaccinated between case-patients and controls (including both control groups) using the equation $\mathrm{VE}=100 \times(1-$ odds ratio $)(1)$. VE over the full surveillance period was assessed, as well as among those with illness onset during March-May and June-July 2021, because of increased circulation of Delta variants in the United States during the latter period (8). Models were adjusted for potential confounders, including admission date (biweekly intervals), U.S. Department of Health and Human Services region, age, sex, and race/ethnicity. Time-varying VE models were then constructed. First, a binary model was constructed by adding a categorical term (2-12 weeks versus 13-24 weeks) for interval from receipt of the second vaccine

\footnotetext{
$\$$ The date of illness onset was used for cases and controls with COVID-19-like illness with median value imputed if missing. For controls without COVID-19like illness, the date of admission minus the median number of days between illness onset and admission for patients with COVID-19 was used for a date of illness onset, also referred to as "illness onset" for this report.
}

dose (among vaccinated participants) and illness onset. Unvaccinated patients were assigned values of zero days since vaccination. In additional analyses, other specifications of time were considered, including using linear and natural cubic spline terms. Bootstrapping with 1,000 replications was used to estimate $95 \%$ CIs. Subgroup analyses included adults aged $\geq 65$ years, patients with immunocompromising conditions, 9 and patients with three or more categories of chronic medical conditions. A sensitivity analysis was also performed including each of the two control groups in models rather than combining them. Significance of association between VE and time since vaccination was assessed using a likelihood-ratio chi-square test with p-values $<0.05$ considered statistically significant. Analyses were conducted using R statistical software (version 4.0.3; R Foundation). This activity was determined to be public health surveillance by each participating site and $\mathrm{CDC}$ and was conducted consistent with applicable federal law and CDC policy.**

After excluding 722 ineligible patients (461 who were not fully vaccinated or unvaccinated, 127 who received a nonmRNA COVID-19 vaccine or mixed vaccines, and 134 who did not meet other inclusion criteria), 3,089 patients were included in the final analysis (1,194 case-patients and 1,895 in the combined control groups) (Table). The median patient age was 59 years (interquartile range $=46-69$ years), $48.7 \%$ were female, $56.7 \%$ were non-Hispanic White, and $21.1 \%$ had an immunocompromising condition. Among case-patients, 141 $(11.8 \%)$ were fully vaccinated as were $988(52.1 \%)$ controls. Among 454 case-patient specimens with SARS-CoV-2 lineage determined, 242 (53.3\%) were identified as Alpha and 74 $(16.3 \%)$ as Delta (Figure 1). Delta variants became the dominant virus in mid-June. Overall VE against hospitalization for COVID-19 was $86 \%(95 \% \mathrm{CI}=82 \%-88 \%)$ over the full surveillance period, including 90\% (95\% CI = 87\%-92\%) among patients without immunocompromising conditions and 63\% (95\% CI $=44 \%-76 \%)$ among patients with immunocompromising conditions. VE among patients with illness onset during March-May was 87\% (95\% CI = 83\%-90\%), and among those with illness onset during June-July was $84 \%$ (95\% CI $=79 \%-89 \%)$. In models considering time since vaccination, $\mathrm{VE}$ was $86 \%(95 \% \mathrm{CI}=82 \%-90 \%)$

\footnotetext{
Immunocompromising conditions included having one or more of the following: active solid organ cancer (active cancer defined as treatment for the cancer or newly diagnosed cancer in the past 6 months), active hematologic cancer (such as leukemia, lymphoma, or myeloma), HIV infection without AIDS, AIDS, congenital immunodeficiency syndrome, previous splenectomy, previous solid organ transplant, immunosuppressive medication, systemic lupus erythematosus, rheumatoid arthritis, psoriasis, scleroderma, or inflammatory bowel disease, including Crohn's disease or ulcerative colitis.

** 45 C.F.R. part 46.102(l)(2), 21 C.F.R. part 56; 42 U.S.C. Sect 241(d); 5 U.S.C. Sect 552a; 44 U.S.C. Sect 3501 et seq.
} 
TABLE. Characteristics of COVID-19 case-patients and controls among hospitalized adults -21 academic medical centers in 18 states, March-July 2021

\begin{tabular}{|c|c|c|c|c|}
\hline \multirow[b]{2}{*}{ Characteristic } & \multicolumn{3}{|c|}{ No. (\%) } & \multirow[b]{2}{*}{ P-value* } \\
\hline & Overall $(\mathrm{N}=3,089)$ & Cases $(n=1,194)$ & Controls $(n=1,895)$ & \\
\hline Median age, yrs (IQR) & $59(46-69)$ & $56(42-66)$ & $62(48-71)$ & $<0.001$ \\
\hline $\begin{array}{l}\text { Age group, yrs } \\
18-49 \\
50-64 \\
\geq 65\end{array}$ & $\begin{array}{r}950(30.8) \\
1,008(32.6) \\
1,131(36.6)\end{array}$ & $\begin{array}{l}445(37.3) \\
424(35.5) \\
325(27.2)\end{array}$ & $\begin{array}{l}505(26.7) \\
584(30.8) \\
806(42.5)\end{array}$ & $<0.001$ \\
\hline $\begin{array}{l}\text { Sex } \\
\text { Female }\end{array}$ & $1,504(48.7)$ & $580(48.6)$ & $924(48.8)$ & 0.921 \\
\hline $\begin{array}{l}\text { Race/Ethnicity } \\
\text { White, non-Hispanic } \\
\text { Black, non-Hispanic } \\
\text { Hispanic, any race } \\
\text { Other, non-Hispanic } \\
\text { Unknown }\end{array}$ & $\begin{array}{r}1,752(56.8) \\
693(22.4) \\
467(15.1) \\
140(4.5) \\
37(1.2)\end{array}$ & $\begin{array}{r}548(45.9) \\
312(26.1) \\
245(20.5) \\
67(5.6) \\
22(1.8)\end{array}$ & $\begin{array}{r}1,204(63.5) \\
381(20.1) \\
222(11.7) \\
73(3.9) \\
15(0.8)\end{array}$ & $<0.001$ \\
\hline $\begin{array}{l}\text { Region } \\
\text { Northeast } \\
\text { South } \\
\text { Midwest } \\
\text { West }\end{array}$ & $\begin{array}{r}432(14.0) \\
1,151(37.3) \\
818(26.5) \\
688(22.3)\end{array}$ & $\begin{array}{l}165(13.8) \\
459(38.4) \\
265(22.2) \\
305(25.5)\end{array}$ & $\begin{array}{l}267(14.1) \\
692(36.5) \\
553(29.2) \\
383(20.2)\end{array}$ & $<0.001$ \\
\hline Resident in long-term care facility (100 unknown) & $141(4.7)$ & $29(2.5)$ & $112(6.1)$ & $<0.001$ \\
\hline Previous hospitalization in last year (231 unknown) & $1,297(45.4)$ & $319(30.0)$ & $978(54.5)$ & $<0.001$ \\
\hline $\begin{array}{l}\text { No. of baseline conditions ( } 2 \text { unknown })^{\S} \\
0 \\
1 \\
2 \\
\geq 3\end{array}$ & $\begin{array}{r}552(17.9) \\
736(23.8) \\
766(24.8) \\
1,033(33.5)\end{array}$ & $\begin{array}{l}301(25.2) \\
310(26.0) \\
260(21.8) \\
322(27.0)\end{array}$ & $\begin{array}{l}251(13.3) \\
426(22.5) \\
506(26.7) \\
711(37.5)\end{array}$ & $<0.001$ \\
\hline $\begin{array}{l}\text { Specific chronic conditions } \\
\text { Cardiovascular disease ( } 1 \text { unknown) } \\
\text { Pulmonary disease (1 unknown) } \\
\text { Diabetes mellitus ( } 1 \text { unknown) } \\
\text { Immunocompromising condition* (2 unknown) }\end{array}$ & $\begin{array}{r}1,900(61.5) \\
804(26.0) \\
952(30.8) \\
652(21.1)\end{array}$ & $\begin{array}{l}647(54.2) \\
257(21.5) \\
348(29.2) \\
205(17.2)\end{array}$ & $\begin{array}{r}1,253(66.2) \\
547(28.9) \\
604(31.9) \\
447(23.6)\end{array}$ & $\begin{array}{r}<0.001 \\
<0.001 \\
0.108 \\
<0.001\end{array}$ \\
\hline Fully vaccinated ${ }^{* *}$ & $1,129(36.6)$ & $141(11.8)$ & $988(52.1)$ & $<0.001$ \\
\hline $\begin{array}{l}\text { Vaccine product received (among fully vaccinated persons) } \\
\text { Pfizer-BioNTech } \\
\text { Moderna } \\
\text { If fully vaccinated, median (IQR) days from second } \\
\text { vaccine dose and onset of symptoms }\end{array}$ & $\begin{array}{r}666(59.0) \\
463(41.0) \\
65(41-93)\end{array}$ & $\begin{array}{r}95(67.4) \\
46(32.6) \\
60(36-94)\end{array}$ & $\begin{array}{l}571(57.8) \\
417(42.2) \\
66(42-93)\end{array}$ & 0.030 \\
\hline
\end{tabular}

\footnotetext{
Abbreviation: $I Q R$ = interquartile range.

* P-values determined using the Wilcoxon rank-sum test for continuous variables and by chi-square test of independence for categorical variables.

${ }^{\dagger}$ Hospitals by region included Northeast: Baystate Medical Center (Springfield, Massachusetts), Beth Israel Deaconess Medical Center (Boston, Massachusetts), Montefiore Medical Center (Bronx, New York); South:Vanderbilt University Medical Center (Nashville, Tennessee), University of Miami Medical Center (Miami, Florida), Emory University Medical Center (Atlanta, Georgia), Johns Hopkins Hospital (Baltimore, Maryland), Wake Forest University Baptist Medical Center (Winston-Salem, North Carolina), Baylor Scott and White Health (Temple, Texas); Midwest: University of lowa Hospitals (lowa City, lowa), University of Michigan Hospital (Ann Arbor, Michigan), Hennepin County Medical Center (Minneapolis, Minnesota), Barnes-Jewish Hospital (St. Louis, Missouri), Cleveland Clinic (Cleveland, Ohio), Ohio State University Wexner Medical Center (Columbus, Ohio); West: Stanford University Medical Center (Stanford, California), UCLA Medical Center (Los Angeles, California), UCHealth University of Colorado Hospital (Aurora, Colorado), Oregon Health \& Science University Hospital (Portland, Oregon), Intermountain Medical Center (Murray, Utah), University of Washington (Seattle, Washington).

$\S$ Chronic condition categories included the following: cardiovascular disease, neurologic disease, pulmonary disease, gastrointestinal disease, endocrine disease, renal disease, hematologic disease, malignancy, immunosuppression not captured in other categories, autoimmune condition, or other condition (sarcoidosis, amyloidosis, or unintentional weight loss $\geq 10$ pounds in the last 90 days).

IImmunocompromising conditions included having one or more of the following: active solid organ cancer (active cancer defined as treatment for the cancer or newly diagnosed cancer in the past 6 months), active hematologic cancer (such as leukemia, lymphoma, or myeloma), HIV infection without AIDS, AIDS, congenital immunodeficiency syndrome, previous splenectomy, previous solid organ transplant, immunosuppressive medication, systemic lupus erythematosus, rheumatoid arthritis, psoriasis, scleroderma, or inflammatory bowel disease, including Crohn's disease or ulcerative colitis.

** COVID-19 vaccination status included unvaccinated, defined as no receipt of any SARS CoV-2 vaccine, and fully vaccinated, defined as receipt of both doses of a 2 -dose mRNA vaccine with the second dose received $\geq 14$ days before illness onset.
} 
during the $2-12$ weeks after the second vaccine dose and $84 \%$ (95\% CI $=77 \%-90 \%) 13-24$ weeks after the second dose; there was no significant difference in VE between these two periods $(\mathrm{p}=0.854)$. Models treating time since vaccination as linear and as a natural cubic spline with a knot at the median and boundary knots at the 10th and 90th percentiles also showed no significant change in VE over a 24-week period (linear $\mathrm{p}=0.400$, spline $\mathrm{p}=0.234$ ) (Supplementary Figure, https://stacks.cdc.gov/view/cdc/108758). No significant change in VE over a 24-week period was observed within subgroups (all $\mathrm{p}>0.05$ ) (Figure 2). In sensitivity analyses, results were similar using individual control groups and combined controls.

\section{Discussion}

In a multistate network that enrolled adults hospitalized during March-July 2021, effectiveness of 2 doses of mRNA vaccine against COVID-19-associated hospitalization was sustained over a follow-up period of 24 weeks (approximately 6 months). These findings of sustained VE were consistent among subgroups at highest risk for severe outcomes from COVID-19, including older adults, adults with three or more

FIGURE 1. Whole genome sequencing lineage determination among adults hospitalized with COVID-19 - 21 academic medical centers in 18 states, $^{*}, \dagger$ March-July 2021

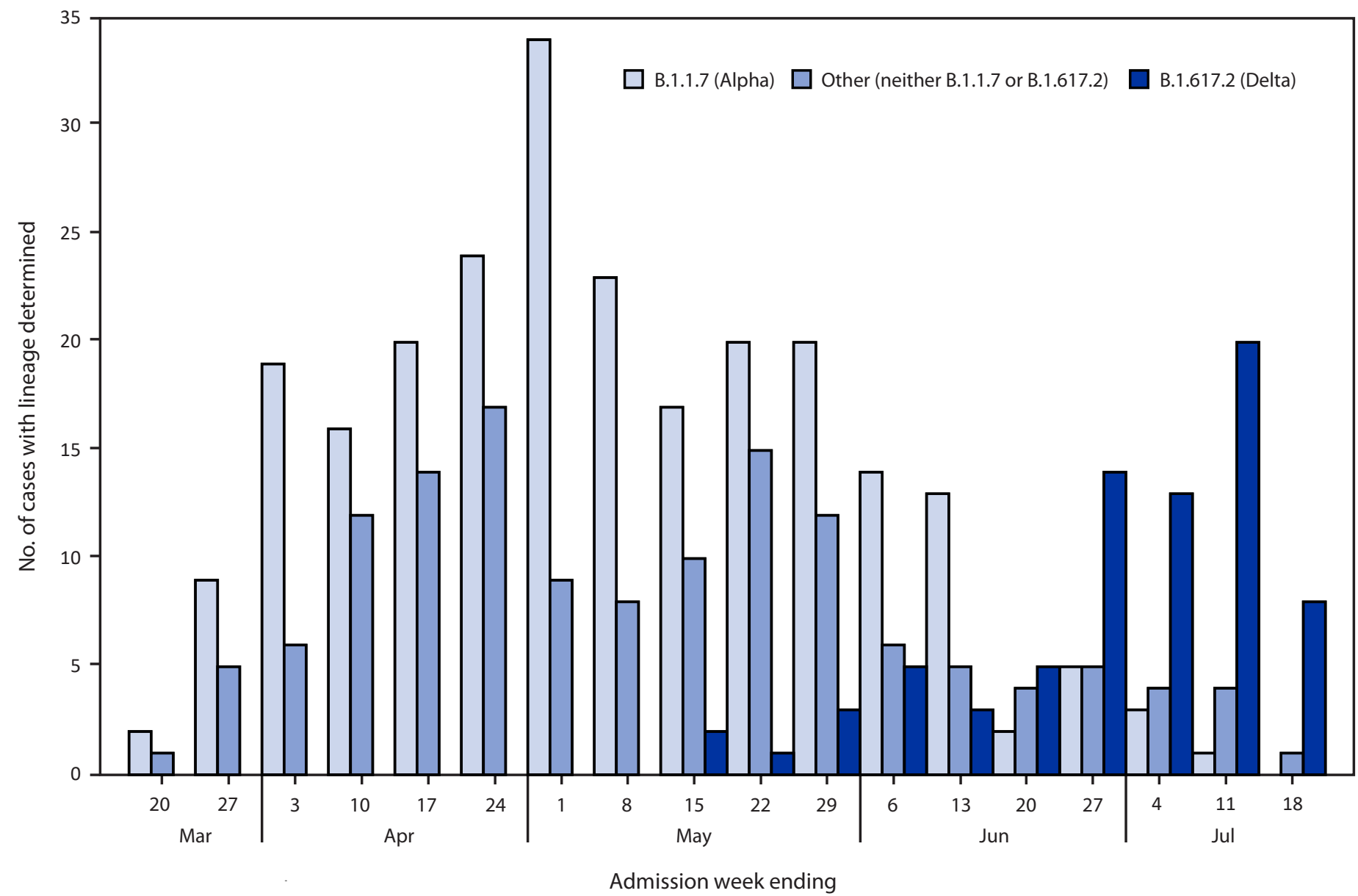

\footnotetext{
* Specimens with SARS-CoV-2 detected by reverse transcription-polymerase chain reaction and with a cycle threshold $<32$ for at least one of two nucleocapsid gene targets tested underwent whole genome sequencing. SARS-CoV-2 lineages were assigned with $>80 \%$ coverage using Pangolin genomes. Results are presented for B.1.1.7 (Alpha) variants, B.1.617.2 (Delta) variants, and other (neither B.1.1.7 or B.1.617.2) variant with lineage determined by whole genome sequencing. Of 74 Delta variants sequenced, four belonged to the AY.3 Delta sublineage. The histogram provides the number of viruses sequenced by week of hospital admission.

† Hospitals by region included Northeast: Baystate Medical Center (Springfield, Massachusetts), Beth Israel Deaconess Medical Center (Boston, Massachusetts), Montefiore Medical Center (Bronx, New York); South:Vanderbilt University Medical Center (Nashville, Tennessee), University of Miami Medical Center (Miami, Florida) Emory University Medical Center (Atlanta, Georgia), Johns Hopkins Hospital (Baltimore, Maryland), Wake Forest University Baptist Medical Center (Winston-Salem, North Carolina), Baylor Scott and White Health (Temple, Texas); Midwest: University of lowa Hospitals (lowa City, lowa), University of Michigan Hospital (Ann Arbor Michigan), Hennepin County Medical Center (Minneapolis, Minnesota), Barnes-Jewish Hospital (St. Louis, Missouri), Cleveland Clinic (Cleveland, Ohio), Ohio State University Wexner Medical Center (Columbus, Ohio); West: Stanford University Medical Center (Stanford, California), UCLA Medical Center (Los Angeles, California), UCHealth University of Colorado Hospital (Aurora, Colorado), Oregon Health \& Science University Hospital (Portland, Oregon), Intermountain Medical Center (Murray, Utah), University of Washington (Seattle, Washington).
} 
chronic medical conditions, and those with immunocompromising conditions. Overall VE in adults with immunocompromising conditions was lower than that in those without immunocompromising conditions but was sustained over time in both populations.

These data provide evidence for sustained high protection from severe COVID-19 requiring hospitalization for up to 24 weeks among fully vaccinated adults, which is consistent with data demonstrating mRNA COVID-19 vaccines have the capacity to induce durable immunity, particularly in limiting the severity of disease $(9,10)$. Alpha variants were the predominant viruses sequenced, although Delta variants became dominant starting in mid-June, consistent with national surveillance data (8). Because of limited sequenced virus, Delta-specific VE was not assessed. VE was similar during June-July when circulation of Delta increased in the United States compared with VE during March-May when Alpha variants predominated, although further surveillance is needed.

The findings in this report are subject to at least six limitations. First, the follow-up period was limited to approximately 24 weeks since receipt of full vaccination because of the recent authorization of mRNA COVID-19 vaccines in the United States. Additional analyses with longer duration of follow-up since vaccination are warranted. Second, effectiveness over time from authorized non-mRNA COVID-19 vaccines, including Janssen's (Johnson \& Johnson) vaccine product, was not assessed because of limited use of this vaccine during the surveillance period. Third, time-varying VE was not assessed

FIGURE 2. Sustained vaccine effectiveness* against COVID-19 among hospitalized adults, by patient status ${ }^{\dagger, \S}$ and interval since vaccination 21 medical centers in 18 states, " March-July 2021

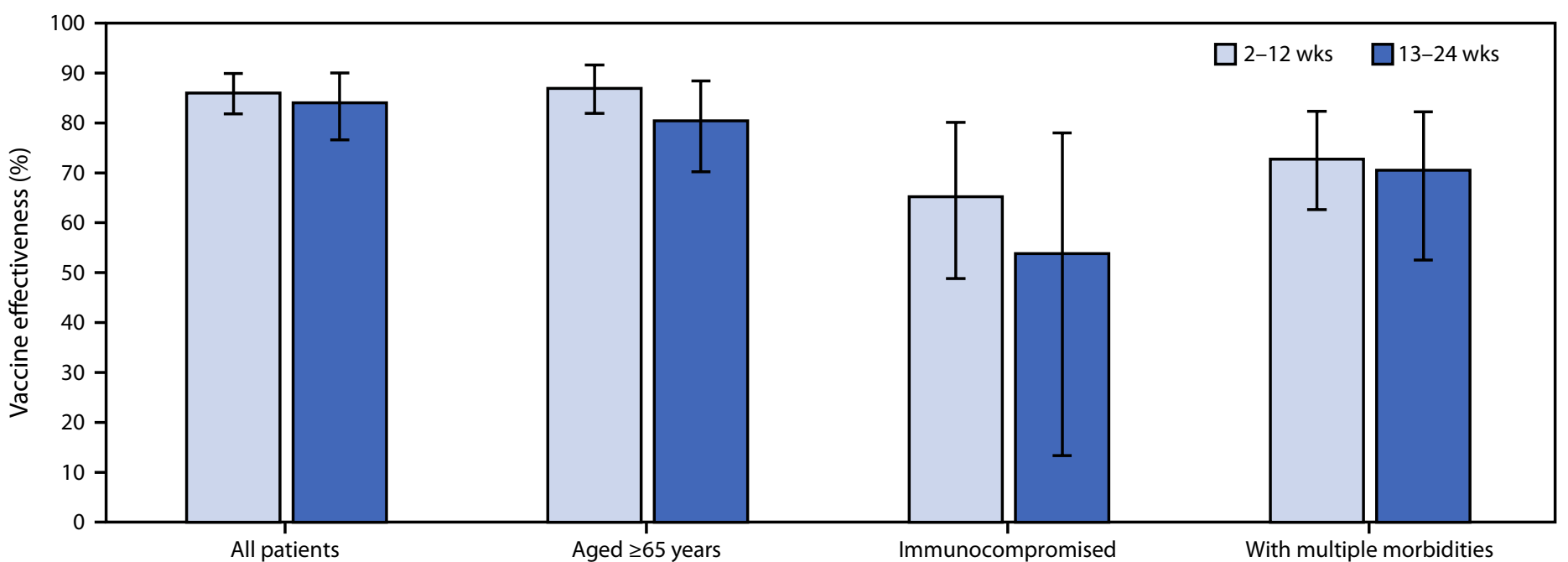

Hospitalized patient status

Abbreviation: $V E=$ vaccine effectiveness.

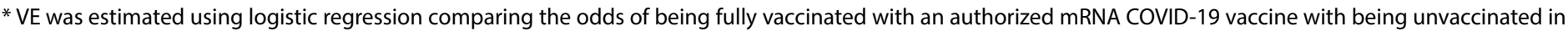

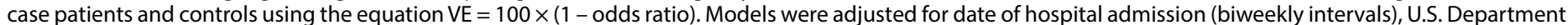

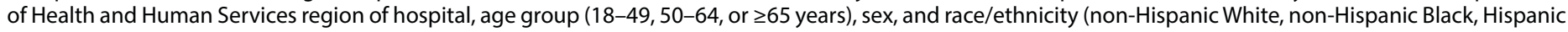

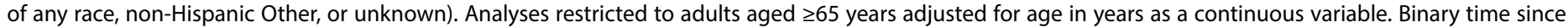
second dose of mRNA vaccine was added to the model with results for 2-12 weeks and 13-24 weeks shown. 95\% confidence intervals shown by error bars.

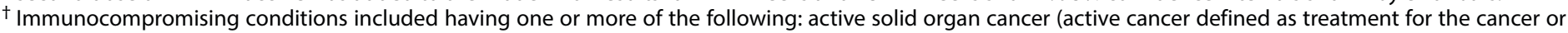

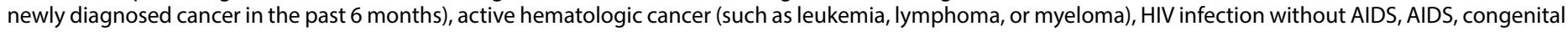

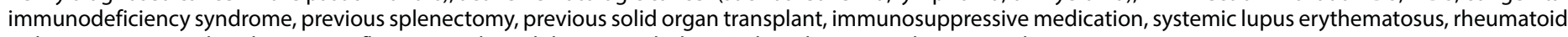
arthritis, psoriasis, scleroderma, or inflammatory bowel disease, including Crohn's disease or ulcerative colitis.

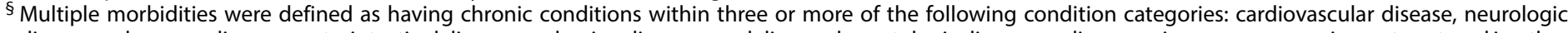

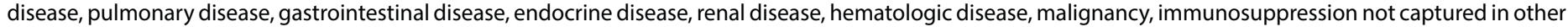
categories, autoimmune condition, or other condition (sarcoidosis, amyloidosis, or unintentional weight loss $\geq 10$ pounds in the last 90 days).

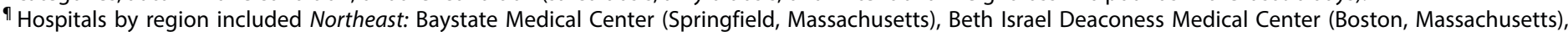

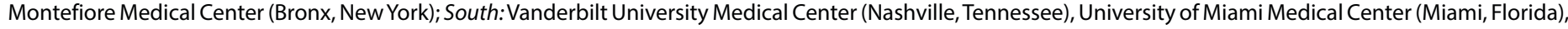

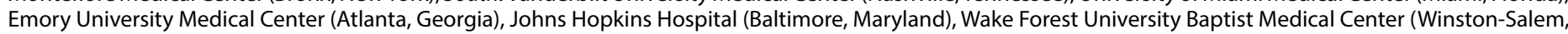

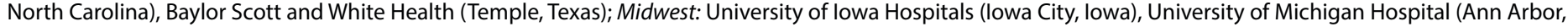

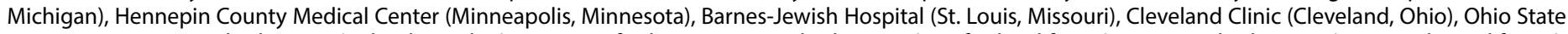

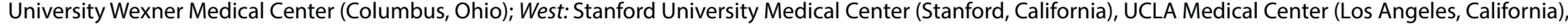

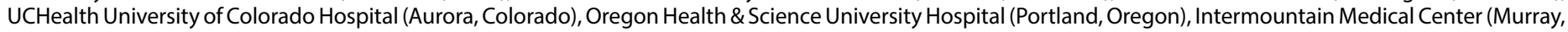
Utah), University of Washington (Seattle, Washington). 


\section{Summary}

What is already known about this topic?

COVID-19 mRNA vaccines provide strong protection against severe COVID-19; however, the duration of protection is uncertain.

What is added by this report?

Among 1,129 patients who received 2 doses of a mRNA vaccine, no decline in vaccine effectiveness against COVID-19 hospitalization was observed over 24 weeks. Vaccine effectiveness was $86 \% 2-12$ weeks after vaccination and $84 \%$ at $13-24$ weeks. Vaccine effectiveness was sustained among groups at risk for severe COVID-19.

What are the implications for public health practice?

mRNA vaccine effectiveness against COVID-19-associated hospitalizations was sustained over 24 weeks; ongoing monitoring is needed as new SARS-CoV-2 variants emerge. To reduce hospitalization, all eligible persons should be offered COVID-19 vaccination.

by lineage because of sample size. Fourth, residual confounding might have been present, although the analysis adjusted for potential confounders, including calendar time and patient age. Fifth, this analysis did not consider VE over time among persons aged $<18$ years or partially vaccinated persons. Finally, the current analysis only included hospitalized adults and did not include persons with asymptomatic SARS-CoV-2 infection or COVID-19 who did not require hospitalization.

Protection against severe COVID-19 resulting in hospitalization was sustained through 24 weeks after vaccination with mRNA COVID-19 vaccines. To reduce their risk for hospitalization, all eligible persons should be offered COVID-19 vaccination. Continued monitoring of VE against infection and severe disease is needed as the elapsed time since vaccination increases and new SARS-CoV-2 variants emerge.

\section{IVY Network}

Nicole Calhoun, Baylor Scott \& White Health; Kempapura Murthy, Baylor Scott \& White Health; Judy Herrick, Baylor Scott \& White Health; Amanda McKillop, Baylor Scott \& White Health; Eric Hoffman, Baylor Scott \& White Health; Martha Zayed, Baylor Scott \& White Health; Michael Smith, Baylor Scott \& White Health; Natalie Settele, Baylor Scott \& White Health; Jason Ettlinger, Baylor Scott \& White Health; Elisa Priest, Baylor Scott \& White Health; Jennifer Thomas, Baylor Scott \& White Health; Alejandro Arroliga, Baylor Scott \& White Health; Madhava Beeram, Baylor Scott \& White Health; Ryan Kindle, Baystate Medical Center; Lori-Ann Kozikowski, Baystate Medical Center; Lesley De Souza, Baystate Medical Center; Scott Ouellette, Baystate Medical Center; Sherell Thornton-Thompson, Baystate Medical Center; Patrick Tyler, Beth Israel Deaconess Medical Center; Omar Mehkri, Cleveland Clinic; Kiran Ashok, Cleveland Clinic; Susan
Gole, Cleveland Clinic; Alexander King, Cleveland Clinic; Bryan Poynter, Cleveland Clinic; Nicholas Stanley, Emory University; Audrey Hendrickson, Hennepin County Medical Center; Ellen Maruggi, Hennepin County Medical Center; Tyler Scharber, Hennepin County Medical Center; Jeffrey Jorgensen, Intermountain Medical Center; Robert Bowers, Intermountain Medical Center; Jennifer King, Intermountain Medical Center; Valerie Aston, Intermountain Medical Center; Brent Armbruster, Intermountain Medical Center; Richard E. Rothman, Johns Hopkins University; Rahul Nair, Montefiore Medical Center; Jen-Ting (Tina) Chen, Montefiore Medical Center; Sarah Karow, Ohio State University; Emily Robart, Ohio State University; Paulo Nunes Maldonado, Ohio State University; Maryiam Khan, Ohio State University; Preston So, Ohio State University; Joe Levitt, Stanford University; Cynthia Perez, Stanford University; Anita Visweswaran, Stanford University; Jonasel Roque, Stanford University; Adreanne Rivera, University of California, Los Angeles; Trevor Frankel, University of California, Los Angeles; Michelle Howell, UCHealth University of Colorado Hospital; Jennifer Friedel, UCHealth University of Colorado Hospital; Jennifer Goff, UCHealth University of Colorado Hospital; David Huynh, UCHealth University of Colorado Hospital; Michael Tozier, UCHealth University of Colorado Hospital; Conner Driver, UCHealth University of Colorado Hospital; Michael Carricato, UCHealth University of Colorado Hospital; Alexandra Foster, UCHealth University of Colorado Hospital; Paul Nassar, University of Iowa; Lori Stout, University of Iowa; Zita Sibenaller, University of Iowa; Alicia Walter, University of Iowa; Jasmine Mares, University of Iowa; Logan Olson, University of Iowa; Bradley Clinansmith, University of Iowa; Carolina Rivas, University of Miami; Hayley Gershengorn, University of Miami; EJ McSpadden, University of Michigan; Rachel Truscon, University of Michigan; Anne Kaniclides, University of Michigan; Lara Thomas, University of Michigan; Ramsay Bielak, University of Michigan; Weronika Damek Valvano, University of Michigan; Rebecca Fong, University of Michigan; William J. Fitzsimmons, University of Michigan; Christopher Blair, University of Michigan; Andrew L. Valesano, University of Michigan; Julie Gilbert, University of Michigan; Christine D. Crider, University of Washington; Kyle A. Steinbock, University of Washington; Thomas C. Paulson, University of Washington; Layla A. Anderson, University of Washington; Christy Kampe, Vanderbilt University Medical Center; Jakea Johnson, Vanderbilt University Medical Center; Rendie McHenry, Vanderbilt University Medical Center; Marcia Blair, Vanderbilt University Medical Center; Douglas Conway, Vanderbilt University Medical Center; Mary LaRose, Wake Forest University; Leigha Landreth, Wake Forest University; Madeline Hicks, Wake Forest University; Lisa Parks, Wake Forest University; Jahnavi Bongu, Washington University; David McDonald, Washington University; Candice Cass, Washington University; Sondra Seiler, Washington University; David Park, Washington University; Tiffany Hink, Washington University; Meghan Wallace, Washington University; Carey-Ann Burnham, Washington University; Olivia G. Arter, Washington University. 
Corresponding author: Mark W. Tenforde, media@cdc.gov.

${ }^{1}$ CDC COVID-19 Response Team; ${ }^{2}$ Vanderbilt University Medical Center, Nashville, Tennessee; ${ }^{3}$ University of Colorado School of Medicine, Aurora, Colorado; ${ }^{4}$ University of Iowa, Iowa City, Iowa; ${ }^{5}$ Baylor Scott $\&$ White Health, Temple, Texas; ${ }^{6}$ Texas A\&M University College of Medicine, Temple, Texas; ${ }^{7}$ Beth Israel Deaconess Medical Center, Boston, Massachusetts; ${ }^{8}$ Wake Forest University Baptist Medical Center, Winston-Salem, North Carolina; ${ }^{9} \mathrm{Johns}$ Hopkins Hospital, Baltimore, Maryland; ${ }^{10}$ Hennepin County Medical Center, Minneapolis, Minnesota; ${ }^{11}$ Montefiore Healthcare Center, Albert Einstein College of Medicine, Bronx, New York; ${ }^{12}$ University of Washington School of Medicine, Seattle, Washington; ${ }^{13}$ Baystate Medical Center, Springfield, Massachusetts; ${ }^{14}$ Intermountain Medical Center and University of Utah, Salt Lake City, Utah; ${ }^{15}$ University of Michigan School of Public Health, Ann Arbor, Michigan; ${ }^{16}$ Oregon Health \& Science University Hospital, Portland, Oregon;

${ }^{17}$ Emory University School of Medicine, Atlanta, Georgia; ${ }^{18}$ Cleveland Clinic, Cleveland, Ohio; ${ }^{19}$ Stanford University School of Medicine, Palo Alto, California; ${ }^{20}$ Ronald Reagan-UCLA Medical Center, Los Angeles, California; ${ }^{21}$ University of Miami, Miami, Florida; ${ }^{22}$ Washington University, St. Louis, Missouri; ${ }^{23}$ Ohio State University Wexner Medical Center, Columbus, Ohio;

${ }^{24}$ University of Michigan School of Medicine, Ann Arbor, Michigan.

All authors have completed and submitted the International Committee of Medical Journal Editors form for disclosure of potential conflicts of interest. Samuel M. Brown reports personal fees from Hamilton, institutional fees from Faron Pharmaceuticals and Sedana, grants from Janssen, the National Institutes of Health (NIH), and the Department of Defense (DoD), book royalties from Oxford University and Brigham Young University, outside the submitted work. Jonathan D. Casey reports grants from NIH, outside the submitted work. Steven Y. Chang was a speaker for La Jolla Pharmaceuticals in 2018 and consulted for PureTech Health in 2020. James D. Chappell reports grants from NIH during the conduct of the study. Matthew C. Exline reports support from Abbott Labs for sponsored talks, outside the submitted work. D. Clark Files reports personal consultant fees from Cytovale and is a data and safety monitoring board (DSMB) member from Medpace, outside the submitted work. Adit A. Ginde reports grants from NIH, DoD, AbbVie, and Faron Pharmaceuticals, outside the submitted work. Michelle N. Gong reports grants from NIH and the Agency for Healthcare Research and Quality (AHRQ), DSMB membership fees from Regeneron, and personal fees from Philips Healthcare, outside the submitted work. Carlos G. Grijalva reports consultancy fees from Pfizer, Merck, and Sanofi-Pasteur; grants from Campbell Alliance/Syneos Health, NIH, the Food and Drug Administration, AHQR, and Sanofi, outside the submitted work. David N. Hager reports salary support from Incyte Corporation, the Marcus Foundation, and EMPACT Precision Medicine via Vanderbilt University Medical Center, outside the submitted work. Natasha Halasa reports grants and nonfinancial support from Sanofi, and Quidel outside the submitted work. Daniel J. Henning reports personal consultant fees from Cytovale and Opticyte. Akram Khan reports grants from United Therapeutics, Johnson \& Johnson, 4D Medical, Lung LLC, and Reata Pharmaceuticals, outside the submitted work. Adam S. Lauring reports personal fees from Sanofi and Roche, outside the submitted work. Christopher J. Lindsell reports grants from NIH, DoD, and the Marcus Foundation; contract fees from bioMerieux, Endpoint LLC, and Entegrion Inc, outside the submitted work and has a patent for risk stratification in sepsis and septic shock issued. Emily T. Martin reports personal fees from Pfizer and grants from Merck, outside the submitted work. Arnold S. Monto reports consulting fees from Sanofi-Pasteur and Seqirus outside the submitted work. Ithan D. Peltan reports grants from NIH and Janssen Pharmaceuticals and institutional support from Asahi Kasei Pharma and Regeneron, outside the submitted work. Todd W. Rice reports personal fees from Cumberland Pharmaceuticals, Inc. and personal fees from Avisa Pharma, LLC and Sanofi, outside the submitted work. Wesley H. Self reports consulting fees from Aeprio Pharmaceuticals and Merck outside the submitted work. No other potential conflicts of interest were disclosed.

\section{References}

1. Tenforde MW, Olson SM, Self WH, et al.; IVY Network; HAIVEN Investigators. Effectiveness of Pfizer-BioNTech and Moderna vaccines against COVID-19 among hospitalized adults aged $\geq 65$ years-United States, January-March 2021. MMWR Morb Mortal Wkly Rep 2021;70:674-9. PMID:33956782 https://doi.org/10.15585/mmwr. $\mathrm{mm} 7018 \mathrm{e} 1$

2. Lopez Bernal J, Andrews N, Gower C, et al. Effectiveness of the PfizerBioNTech and Oxford-AstraZeneca vaccines on covid-19 related symptoms, hospital admissions, and mortality in older adults in England: test negative case-control study. BMJ 2021;373:n1088. PMID:33985964 https://dx.doi.org/10.1136\%2Fbmj.n1088

3. Haas EJ, Angulo FJ, McLaughlin JM, et al. Impact and effectiveness of mRNA BNT162b2 vaccine against SARS-CoV-2 infections and COVID-19 cases, hospitalisations, and deaths following a nationwide vaccination campaign in Israel: an observational study using national surveillance data. Lancet 2021;397:1819-29. PMID:33964222 https:// doi.org/10.1016/S0140-6736(21)00947-8

4. Tenforde MW, Patel MM, Ginde AA, et al. Effectiveness of SARS-CoV-2 mRNA vaccines for preventing Covid-19 hospitalizations in the United States. Clin Infect Dis. In press 2021.

5. Thompson MG, Burgess JL, Naleway AL, et al. Prevention and attenuation of Covid-19 with the BNT162 b2 and mRNA-1273 vaccines. N Engl J Med 2021;385:320-9. PMID:34192428 https://doi. org/10.1056/NEJMoa2107058

6. Ferdinands JM, Gaglani M, Martin ET, et al. Waning vaccine effectiveness against influenza-associated hospitalizations among adults, 2015-2016 to 2018-2019, US Hospitalized Adult Influenza Vaccine Effectiveness Network. Clin Infect Dis 2021. Epub January 19, 2021. PMID:33462610 https://doi.org/10.1093/cid/ciab045

7. Feng S, Chiu SS, Chan ELY, et al. Effectiveness of influenza vaccination on influenza-associated hospitalisations over time among children in Hong Kong: a test-negative case-control study. Lancet Respir Med 2018;6:925-34. PMID:30442587 https://doi.org/10.1016/ S2213-2600(18)30419-3

8. CDC. COVID data tracker. Atlanta, GA: US Department of Health and Human Services, CDC; 2021. Accessed August 5, 2021. https:// covid.cdc.gov/covid-data-tracker/\#datatracker-home

9. Turner JS, O'Halloran JA, Kalaidina E, et al. SARS-CoV-2 mRNA vaccines induce persistent human germinal centre responses. Nature 2021;596:109-13. PMID:34182569 https://doi.org/10.1038/ s41586-021-03738-2

10. Cromer D, Juno JA, Khoury D, et al. Prospects for durable immune control of SARS-CoV-2 and prevention of reinfection. Nat Rev Immunol 2021;21:395-404. PMID:33927374 https://doi.org/10.1038/ s41577-021-00550-x 